Journal of Applied Fluid Mechanics, Vol. 3, No. 2, pp. 33-41, 2010.

Available online at www.jafmonline.net, ISSN 1735-3572, EISSN 1735-3645.

DOI: $10.36884 / \mathrm{jafm} .3 .02 .11886$

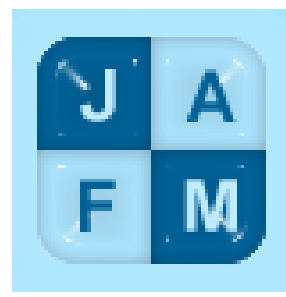

\title{
Slip-Flow and Heat Transfer in Isoflux Rectangular Microchannels with Thermal Creep Effects
}

\author{
H. Niazmand ${ }^{1}$, A. Amiri Jaghargh ${ }^{1}$ and M. Renksizbulut ${ }^{2}$ \\ ${ }^{1}$ Ferdowsi University of Mashhad, Mechanical Engineering Department, Mashhad, Iran \\ ${ }^{2}$ University of Waterloo, Mechanical \& Mechatronics Engineering Department, \\ Waterloo, Ontario, Canada, N2L $3 G 1$ \\ Email: hniazmand@yahoo.com
}

(Received November 15, 2008; accepted July 12, 2009)

\begin{abstract}
A control-volume numerical approach has been used to study rarefaction effects in simultaneously hydrodynamically and thermally developing flow in rectangular microchannels with a prescribed uniform wall heat flux in the slip-flow regime $\left(10^{-3} \leq \mathrm{Kn} \leq 10^{-1}\right)$. The effects of velocity slip and thermal creep on the key flow parameters are examined in detail. Low Reynolds number flows $(\operatorname{Re} \leq 1)$ for different channel aspect ratios $\left(0 \leq \alpha^{*} \leq 1\right)$ are considered. The effects of rarefaction on the global features of the flow and thermal development in the entrance region are examined. Dramatic reductions in the friction coefficient are observed in the entrance region due to rarefaction effects, which are enhanced by thermal creep. For the fluid heating cases considered here, thermal creep increases slip at the wall and thereby further reduces the friction coefficient and slightly enhances heat transfer at a given Reynolds number. For an identical heat flux applied to the microchannel walls, thermal creep effects become much more pronounced at lower Reynolds numbers since it results in higher axial temperature gradients.
\end{abstract}

Keywords: Rectangular microchannel, slip-flow, thermal creep, entrance region, constant heat flux

\section{INTRODUCTION}

Fluid flow and heat transfer at micro scale have become a leading area of research due to the rapid growth of MEMS applications in medical and biological systems, fuel cell technology, microactuators, microsensors and microchannel heat sinks among others. Microchannels as one of the key components of many of these devices require the analysis of gas flows through micron-size conduits.

An important effect associated with gas flows in microchannels is the rarefaction effect. Rarefaction occurs when the molecular mean free path $(\lambda)$ of the flowing medium has the same order of magnitude as the channel size. This can happen in microchannels where the characteristic length scale of the conduit is small. The Knudsen number ( $\mathrm{Kn})$, which is defined as the ratio of $\lambda$ to the length scale of the flow, is a measure of rarefaction. For Knudsen numbers in the range $10^{-3}<$ $\mathrm{Kn}<10^{-1}$, which is called the slip-flow regime, the flow does not obey the classical continuum physics where the no-slip condition is valid. In fact, as the Kn number increases the portion of molecular collisions with walls incrementally dominate over intermolecular collisions which leads to a deviation from thermodynamic equilibrium and the continuum concept. It is well established that in these flows the standard NavierStokes and energy equations are still valid if accompanied by appropriate velocity-slip and temperature-jump boundary conditions.

In heat-sink applications of microchannels, a constant heat flux is usually applied to the microchannel walls leading to different temperature boundary conditions depending on the conductivity of the components. The boundary conditions which are mostly referred to in the literature are $\mathrm{H} 1$ and $\mathrm{H} 2$ according to Shah and London (1978). The former assumes a uniform axial heat flux, while the wall temperature at each cross section is constant. The latter, which is the case of present study, presumes a uniform heat flux both axially and peripherally allowing for the wall temperature to vary circumferentially.

Slip-flow heat transfer in microchannels has been the subject of many studies in the past. Spiga and Morini (1998) investigated developing heat transfer in rectangular microchannels of different aspect ratios. They reported developing thermal behavior and Nusselt numbers for different combinations of heated and adiabatic walls. Kuddusi and Cetegen (2007) developed a mathematical model to study thermally developed heat transfer in rectangular microchannels with constant 
heat flux boundary conditions. Nusselt numbers for a wide range of Knudsen numbers and aspect ratios were reported for different combinations of heated and adiabatic walls. Hooman (2008) developed an analytical approach to investigate fully developed (both hydrodynamically and thermally) flows in microchannels of arbitrary cross section under uniform heat flux boundary conditions. Cetin et al. (2008) numerically solved the developing temperature field in hydrodynamically fully developed microtubes considering axial conduction and viscous dissipation. Developing slip-flow and heat transfer in trapezoidal microchannels under constant wall temperature was investigated numerically by Niazmand et al. (2008). They reported developing friction coefficient and Nusselt number for a wide range of Knudsen numbers and aspect ratios. In addition, different correlations were proposed for fully developed friction and heat transfer coefficients.

Another effect related to the rarefied gas flows is the thermal creep phenomenon, which contributes to velocity slip along the surface due to a tangential temperature gradient in the gas layer adjacent to the wall. For cases with sufficiently high axial temperature gradients at the walls, thermal creep can considerably influence the flow field. This is especially true for very low Reynolds number flows with high heat fluxes at the boundaries, which is the case for many microfluidic heat-sink applications.

A survey of the available literature shows a limited number of studies on thermal creep effects in microchannels. Rij et al. (2007) numerically studied fluid flow and heat transfer in the entrance region of a planar microchannel. Both thermally and hydrodynamically developing slip-flows with prescribed constant creep velocities were investigated. They also presented an analytical solution for fully developed flow. Sazhin et al. (2008) studied the thermal creep phenomenon through straight cylindrical capillaries in the free molecular flow regime using the DSMC method. Méolans and Graur (2008) developed an analytical model for thermal creep flow. They considered a planar microchannel between two reservoirs maintained at the same pressure, while a constant temperature gradient was applied along the channel. Bao and Lin (2008) used the Burnett equations with slip boundary conditions to model the compressible gas flow and heat transfer in micro Poiseuille flow in slip and transition flow regimes. The thermal creep contribution on the slip velocity was taken into consideration in the simulation. They concluded that thermal creep affects the slip velocity only in the entrance region where high tangential temperature gradients occur. An analytical solution for the velocity profile of the compressible Poiseuille flow with the thermal creep effect and a constant heat flux boundary condition is derived by Ghahremani et al. (2008). Using a perturbation method, they concluded that the thermal creep increases the velocity and the flow rate for a heating case. It was also shown that this effect is only considerable for lower orders of magnitude of the Brinkman number $(\sim 0.01)$.
In the present study, incompressible gaseous slip-flow and heat transfer in the entrance region of rectangular microchannels of various aspect ratios are investigated for a prescribed constant heat flux boundary condition. Three-dimensional Navier-Stokes and energy equations along with velocity-slip and temperature-jump boundary conditions are solved numerically by a control-volume method. The effects of thermal creep and axial heat conduction are included in the analysis.

\section{FORMULATIONS}

The flow geometry is as shown in Fig. 1. The coordinate system is located in the center of the channel and the aspect ratio is denoted by $\alpha^{*}=2 h / 2 a$.

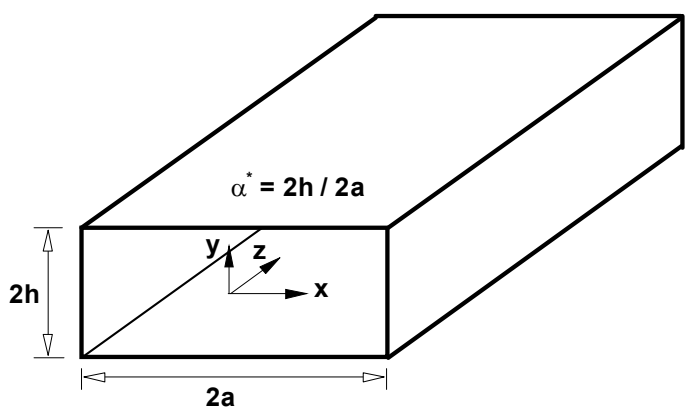

Fig. 1. Flow geometry and the coordinate system

The hydraulic diameter of the channel is set to unity and dimensions of the channel are calculated based on the channel aspect ratio and definition of hydraulic diameter as $D_{h}=4(a h) /(a+h)$. Such a setting provides a more convenient base for the comparison of results since $D_{h}$ is used as a length scale for nondimensionalization. Standard integral forms of continuity, momentum and energy equations for constant-property laminar flows are used in modeling the fluid flow and thermal field as follows:

$$
\begin{aligned}
& \int_{A} \vec{V} \cdot d \vec{A}=0 \\
& \frac{\partial}{\partial t} \int_{\forall} \rho \vec{V} d \forall+\int_{\forall} \rho \vec{V} \cdot \vec{\nabla} \vec{V} d \forall= \\
& -\int_{A} p d \vec{A}+\int_{A} \mu \vec{\nabla} \vec{V} \cdot d \vec{A} \\
& \frac{\partial}{\partial t} \int_{\forall} \rho c_{p} T d \forall+\int_{\forall} \rho c_{p} \vec{V} \cdot \vec{\nabla} T d \forall=\int_{A} k \vec{\nabla} T \cdot d \vec{A}
\end{aligned}
$$

where $\vec{V}, T, \rho, p, \mu, c_{p}$ and $k$ are the velocity vector, temperature, density, pressure, dynamic viscosity, specific heat and thermal conductivity, respectively.

Inflow boundary conditions correspond to uniform flat profiles such that $w=W_{m}=W_{i}$ and $T=T_{i}$, where subscripts $i$ and $m$ refer to inlet and bulk mean conditions, respectively. For outflow, zero-gradients along the axial flow direction are applied for velocity. For a constant heat flux condition, the axial variation of temperature is linear outside the entrance region, and therefore, a linear extrapolation is used to determine the 
temperature at the outlet. The pressure is set to zero at the outlet while zero pressure gradients are assigned at all other boundaries including the inlet for the massdriven flow considered in the present work. The flow satisfies the velocity-slip and temperature-jump conditions at the walls, given by:

$$
\begin{aligned}
& w_{s}=\left(\frac{2-\sigma_{v}}{\sigma_{v}}\right) \lambda\left(\frac{\partial w}{\partial n}\right)_{g}+\frac{3}{4} \frac{\mu}{\rho T_{g}}\left(\frac{\partial T}{\partial s}\right)_{g} \\
& T_{g}-T_{w}=\left(\frac{2-\sigma_{T}}{\sigma_{T}}\right)\left(\frac{2 \gamma}{\gamma+1}\right) \frac{\lambda}{\operatorname{Pr}}\left(\frac{\partial T}{\partial n}\right)_{g}
\end{aligned}
$$

The other velocity components are specified similarly. Here, $w_{s}$ is the slip velocity defined as $w_{s}=w_{g}-w_{w}$, where $w_{g}$ represents the gas velocity on a wall and $w_{w}$ is the wall velocity. The subscript $w$ identifies a wall with a normal coordinate $n$ and tangential coordinate $s$. The subscript $g$ indicates the first layer of the gas adjacent to the wall where the normal and tangential temperature gradients are evaluated. The coefficients $\sigma_{v}$ and $\sigma_{T}$, known as the tangential-momentum and energy accommodation coefficients, are usually determined experimentally. They are taken as unity in the present study. It must be emphasized that thermal creep is only considered in the axial flow direction.

\section{Numerical Method}

Using the control-volume method, the governing equations are integrated over corresponding finite volumes while they are transformed in a generalized non-orthogonal coordinate system. The numerical solution is based on a projection-type method that solves the momentum equations in two steps. First, an intermediate velocity field is obtained using the available pressure field. Next, velocity and pressure corrections are calculated from a Poison equation obtained from the continuity equation. The numerical scheme was originally developed by Chorin (1968), and improved further by Dwyer (1989) and the present authors $(2006,2008)$. Also, a pressure correction based on the conservation of cross sectional mass flux is introduced, which greatly enhances the convergence rate of the numerical scheme. A pressure defect for a given cross section is associated with the average velocity defect $\Delta w^{\prime}$ at the same cross section according to the following equation:

$\rho \frac{\Delta w^{\prime}}{\Delta t}=-\frac{\partial p^{\prime}}{\partial x}$

The average velocity defect is defined as $\Delta w^{\prime}=\bar{W}-W_{i} \quad$ where $\bar{W}$ is the predicted cross sectional average velocity. Thus, the pressure filed is updated through the above correction and then the new velocity field is obtained using the updated pressure field.

\section{GRID INDEPENDENCE AND VALIDATION}

Numerous computations were performed to study the required cross sectional and axial grid resolutions. The effect of cross sectional grid resolution on some key flow parameters is studied in Fig. 2 for flow in a square microchannel. The variation of less than one percent present in friction and heat transfer coefficients for cross sectional mesh size beyond $51 \times 51$ is an indication of satisfactory grid resolution. The effect of axial grid resolution on apparent friction factor for a fixed cross sectional grid of $51 \times 51$ is studied in Fig. 3. The axial variation of the apparent friction coefficient is almost unaffected by the increment of axial grid points further than 141 points. Therefore a minimum mesh size of $51 \times 51 \times 141(x, y, z)$ with grid expansion ratios of about 1.06, 1.1 and 1.04 in the $x, y$ and $z$ directions, respectively, have been employed in the numerical computations.

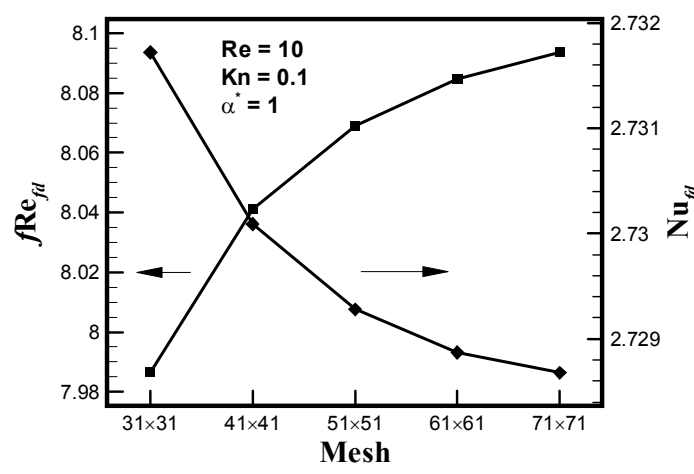

Fig. 2. Effects of cross sectional resolution on friction and heat transfer coefficients.

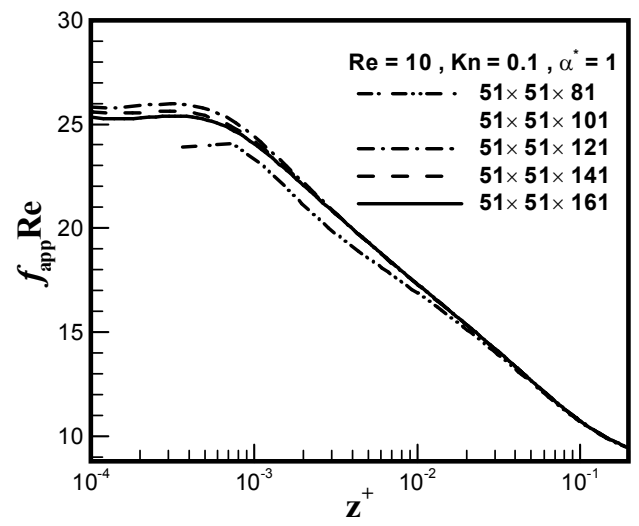

Fig. 3. Effect of axial grid distribution on apparent friction factor.

Morini et al. (2004) have numerically investigated the rarefaction effects on the pressure drop for a fully developed laminar incompressible flow through silicon microchannels having rectangular and trapezoidal cross sections in the slip-flow regime. To validate the present numerical code, the fully developed friction coefficients are compared with their results at various Knudsen numbers and for three different aspect ratios as shown in Fig. 4. 
Furthermore, the fully-developed heat transfer coefficients for a constant heat flux condition have been compared with the analytical results of Tunc and Bayazitoglu (2002) and Kuddusi and Cetegen (2007) for incompressible fully developed square micochannel flows in Fig. 5. The slightly higher numerical predictions of the present study as compared to the analytical results can be attributed to the inclusion of axial heat conduction, which is important for low Reynolds number flows associated with microchannel flows.

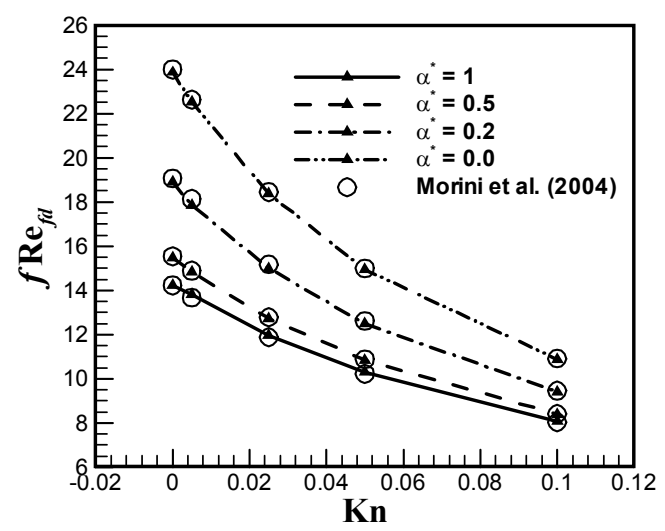

Fig. 4. Comparison of fully developed friction factor for various aspect ratios and Kn numbers.

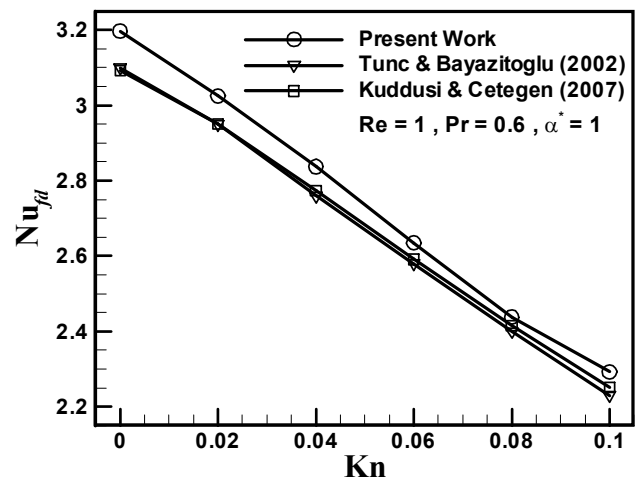

Fig. 5. Comparison of fully developed Nu number for wide range of Knudsen numbers.

For thermal creep, much less information is available. Therefore, the fully developed friction and heat transfer coefficients in planar-microchannel flows are compared with the analytical results of Rij et al. (2007) in Fig. 6 where excellent agreement is observed.

\section{Results AND Discussions}

The Reynolds number, $\operatorname{Re}=\rho W_{m} D_{h} / \mu$, is based on the uniform inlet velocity and the hydraulic diameter. The density is constant, therefore, $W_{m}=W_{\mathrm{i}}$ everywhere in the channel. Present study is concentrated on low Re flows, $(\operatorname{Re} \leq 1)$. The Knudsen number, defined as $\mathrm{Kn}=\lambda / D_{h}$, varies in the range of $0 \leq \mathrm{Kn} \leq 10^{-1}$.

In the present study, the Prandtl number is set equal to 1 and therefore the nondimensional axial length is the same for momentum and heat transfer equations, $z^{+}=$ $z /\left(D_{h} \mathrm{Re}\right)=z /\left(D_{h} \mathrm{PrRe}\right)$. The non-dimensional heat flux, $q=q^{\prime \prime} D_{h} / k T_{i}$, is prescribed as $q=0.007$ unless otherwise stated.

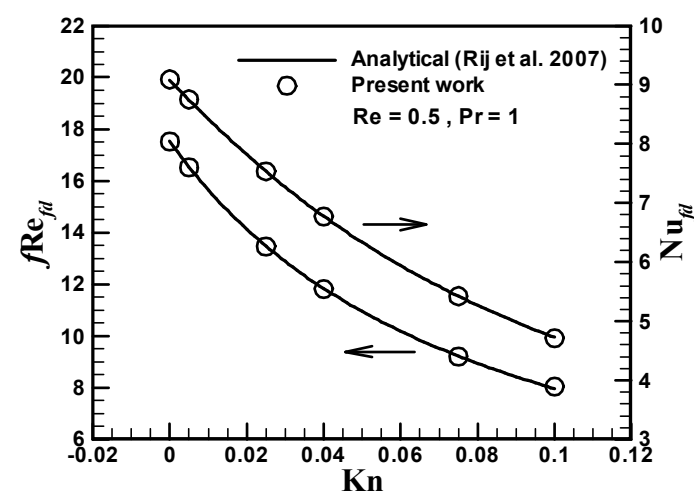

Fig. 6. Comparison of fully developed friction and heat transfer coefficients in the presence of thermal creep.

\subsection{The Flow Field}

In general, velocity profile in the slip-flow regime is affected by normal velocity and axial temperature gradients. For mass driven flows with a prescribed uniform inlet velocity both gradients are strong at the inlet. As indicated by Eq. (4), thermal creep effects become smaller as the temperature of the gas layer adjacent to the wall increases. For a microchannel with constant heat flux, it is expected that thermal creep effects lose their strength far from the inlet where the axial temperature gradient becomes almost constant while the fluid temperature increases axially. However, close to the inlet the relative importance of thermal creep to the slip contribution from the normal velocity gradients is dependent on the applied heat flux and Re. For an identical heat flux applied to the microchannel walls, thermal creep effects become more pronounced at lower Reynolds numbers since this results in higher axial temperature gradients at the wall. Therefore, different flow patterns can be obtained close to the inlet depending on the values of Re and the applied heat flux.

Present results indicate that thermal creep does not influence the flow field significantly at $\operatorname{Re}=1$ with the considered applied heat flux. However, at lower Re, thermal creep can affect the flow pattern considerably, especially close to the channel inlet, as shown in Fig. 7 where the axial velocity contours are plotted in the symmetry plane $(x=0)$ for (a) $\mathrm{Re}=1$ and (b) $\mathrm{Re}=0.1$ in a square microchannel. Clearly, the developing flow pattern at $\operatorname{Re}=0.1$ is much different from that for $\mathrm{Re}=$ 1. Two distinct regions can be identified for the $R e=0.1$ case. Close to the inlet, thermal creep effects are dominant due to high axial temperature gradients and low fluid temperature at wall, and therefore, the region of high velocity is near the wall such that the uniform inlet velocity profile transforms into a parabola with the minimum velocity in the core region and maximum velocity at the wall. This is clearly seen in Fig. 8 where the evolutions of the axial velocity profiles are plotted at different axial locations. For further clarity, different stages in the profile development are shown separately 
in Fig. 8. An increase of about $12 \%$ occurs in the axial velocity component from the uniform inlet velocity near the wall due to strong slip effects at the axial
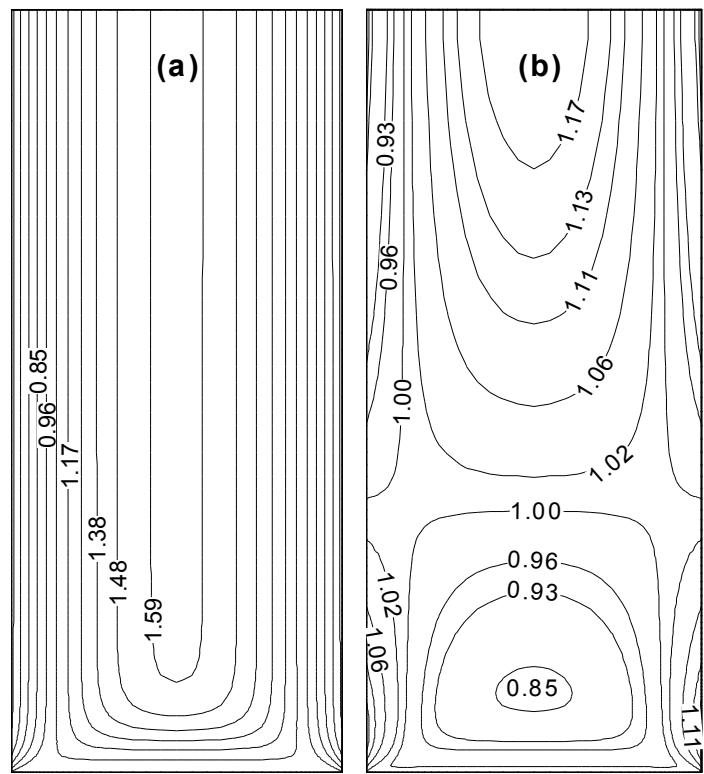

Fig. 7. Axial velocity contours in the symmetry plane of a square microchannel for $\mathrm{Kn}=0.1$ and (a) $\operatorname{Re}=1$, (b) $\operatorname{Re}=0.1$

location of $z^{+}=5.162$ shown in Fig. 8a. However, farther down stream, where the axial temperature gradient approaches a constant value, thermal creep effects lose their strength and velocity profile converts from the inverted parabola to a flat profile (Fig. $8 \mathrm{~b}, \mathrm{z}^{+}=$ 17.21), which latter transforms into the conventional parabolic velocity profile with a large velocity slip of about $84 \%$ of the inlet velocity (Fig. $8 \mathrm{c}, \mathrm{z}^{+}=50$ ).

In Fig. 9, three dimensional streamwise velocity profiles for $\mathrm{Re}=0.1$ and $\mathrm{Kn}=0.1$ are shown at several axial locations for cases with and without thermal creep. This figure shows the three dimensional structures of different velocity profiles in their deformation from a uniform inlet profile to their fully developed form. These velocity profiles, which are self explanatory, are cut in their symmetry plane for clarity. The dimensionless pressure drop along the channel is expressed in the form of an apparent friction factor defined as:

$$
f_{\text {app }} \operatorname{Re}=\frac{1}{4 z^{+}} \frac{\Delta \bar{p}}{\rho W_{i}^{2} / 2}
$$

where $\Delta \bar{p}$ indicates the pressure drop from the entrance. In addition to the wall shear stresses, which are the only sources of the pressure drop in fully developed region, changes in the momentum rate account for the major portion of the pressure drop in the entrance region.
Figure 10 shows the variation of the apparent friction coefficient with $\mathrm{Kn}$ at $\mathrm{Re}=1$ along the channel. For comparison, cases with thermal creep are identified with a circle in this figure. Major reduction in the apparent friction coefficient in the entrance region can be attributed to two factors. Slip reduces the wall shear stresses and at the same time less pressure drop is required for transforming the velocity profile from a uniform inlet profile to a fully developed profile (since the fully developed velocity profiles are much flatter as compared to those with the no-slip condition). These two factors result in lower friction coefficients at higher $\mathrm{Kn}$
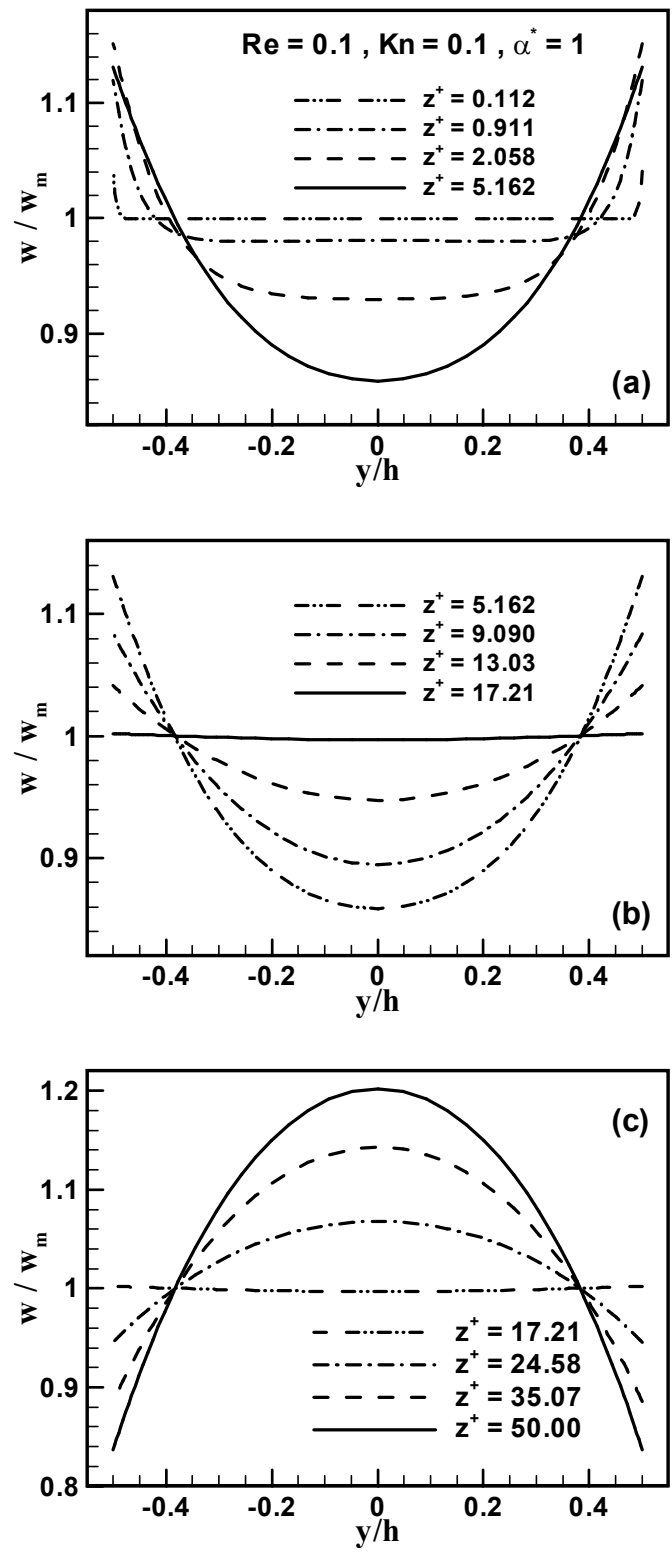

Fig. 8. Effects of thermal creep on axial velocity profiles in a square microchannel at $\operatorname{Re}=0.1$, $\mathrm{Kn}=0.1$ and different axial locations. 

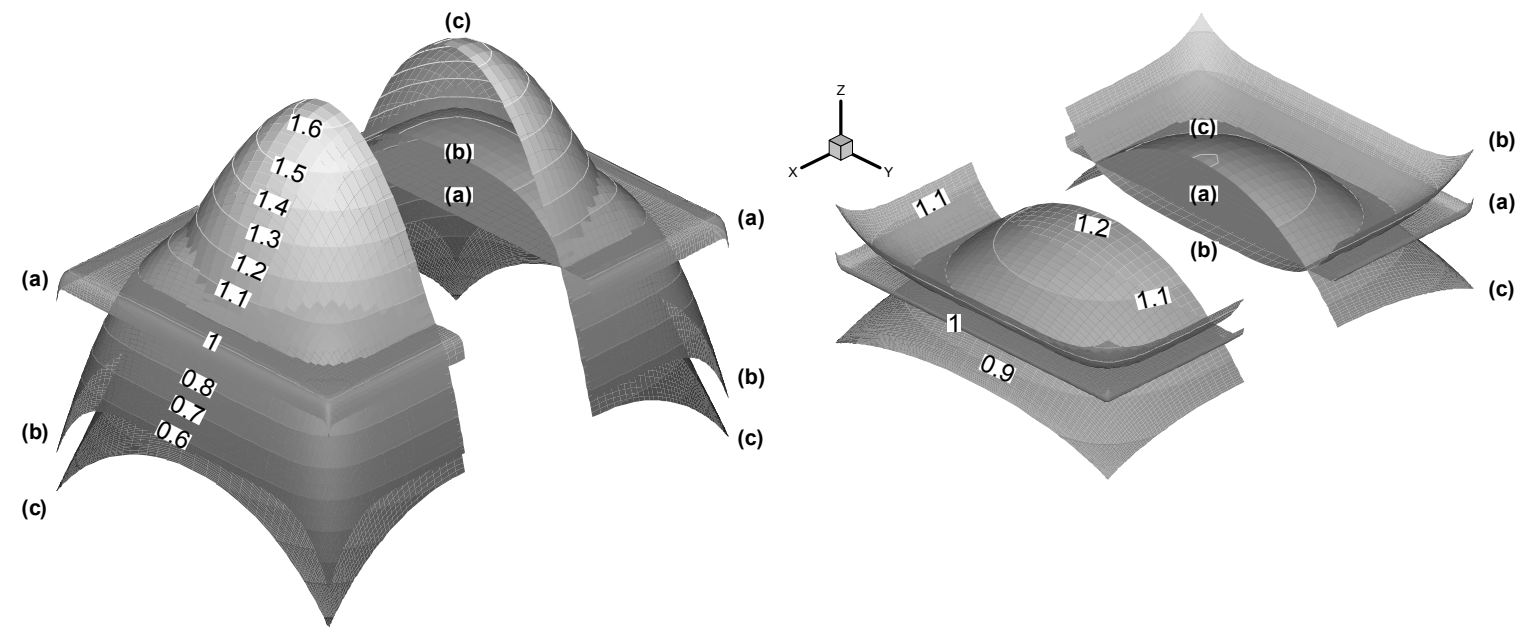

Fig. 9. 3-D streamwise velocity profiles for $\mathrm{Re}=0.1$ and $\mathrm{Kn}=0.1$

LHS: without thermal creep; RHS: with thermal creep.
(a) $\mathrm{z}^{+}=0.0112$
(b) $\mathrm{z}^{+}=2.058$
(c) $\mathrm{z}^{+}=50$

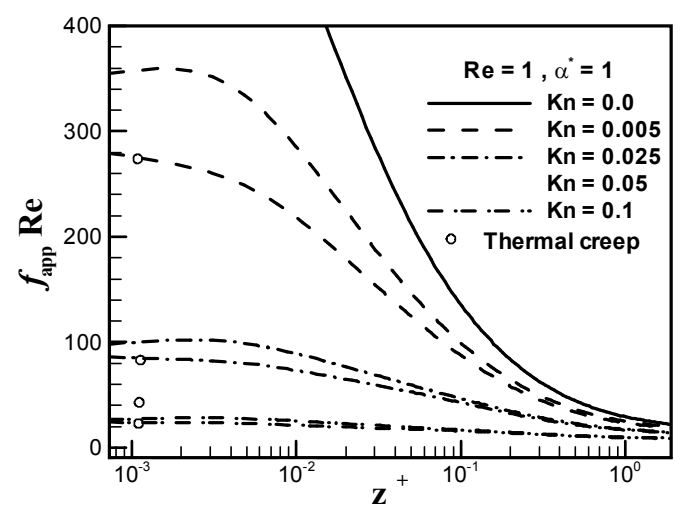

Fig. 10. Axial variation of apparent friction coefficient with and without thermal creep.

numbers. Both factors are slightly enhanced when the contribution of thermal creep is considered. The change of fully developed $f \operatorname{Re}$ for variation of $\mathrm{Kn}$ number from 0.0 to 0.1 at $\operatorname{Re}=1$ is about $44 \%$ in a square duct, while this value increases to $48 \%$ for a duct with aspect ratio of 0.2 . Thermal creep further reduces the fully developed friction coefficients by almost $2 \%$ for $\mathrm{Kn}=0.1$ in a square duct at $\mathrm{Re}=1$.

\subsection{The Temperature Field}

The temperature field in rarefied flows is a function of geometry, Re, Pr and Kn numbers. For the prescribed constant heat flux boundary condition, a dimensionless temperature can be defined as:

$\theta=\frac{\left|T-T_{r e f}\right|}{q^{\prime \prime} D_{h} / k}$

where $T_{r e f}$ is a reference temperature. The nondimensional temperature profile can reveal different features depending on the choice of the reference temperature as will be discussed later. In Fig. 11, cross- sectional temperature distributions are compared for the slip and no-slip cases at $\mathrm{Re}=1$. Three different axial locations in a square microchannel are considered. For this case, the inlet temperature $T_{i}$ is taken as the reference temperature and the dashed lines on the right hand side indicate the slip case with $\mathrm{Kn}=0.1$ while the solid lines refer to the no-slip case. This figure indicates almost similar temperature distributions for both the slip and no-slip-flows close to the entrance; however, in the fully developed region the core is slightly warmer in the slip case as compared to the no-slip case.

A better view of the entrance thermal development can be obtained through the axial variations of the temperature profiles in the symmetry plane as shown in Fig. 12. In this figure, the local wall temperature has been used as $T_{r e f}$. Note that using $T_{i}$ as the reference temperature in the definition of the non-dimensional temperature cannot reveal the temperature jump at the wall. Furthermore, non-dimensional temperature profiles based on $T_{i}$ as reference temperature do not lead to a fixed profile in the fully developed region. Using local wall temperature as the reference temperature provides a base to present the wall temperature jump and leads to a fixed profile in the fully developed region. The only drawback of such a choice is that the reference temperature is not constant across the temperature field.

The effects of thermal creep on the temperature profiles are also shown in Fig. 12, where on the right half of the temperature profiles (shown by dashed lines) the thermal creep effect is included, while on the left side (shown by dotted lines) thermal creep is ignored. Despite the variation in the temperature profiles corresponding to their axial locations in the symmetry plane, the temperature jump is identical at all axial locations. This is due to the fact that local wall temperature has been used as the reference temperature along with the constant heat flux boundary condition. 

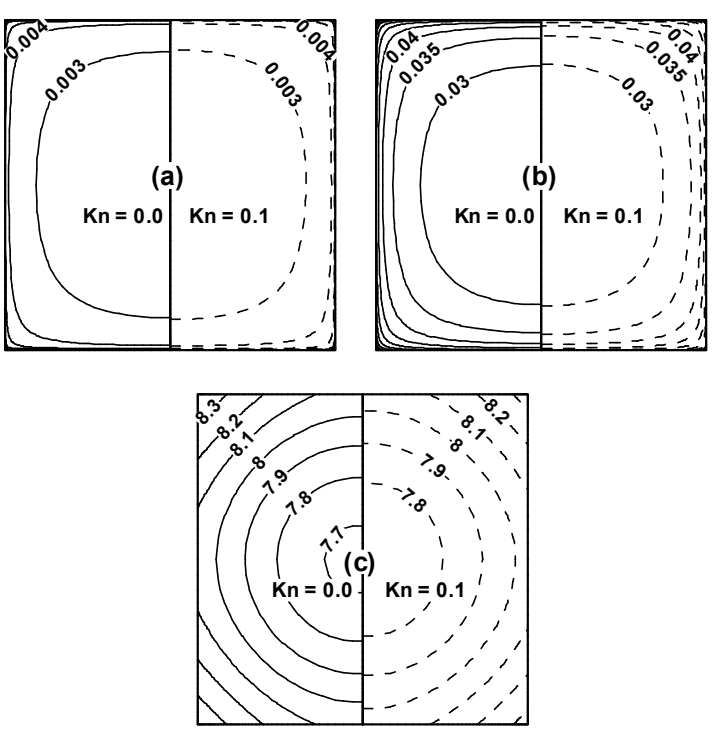

Fig. 11. Cross-sectional temperature distributions for flow in a square duct at $\operatorname{Re}=1$. Solid lines: $K n=0$; dashed lines: $\mathrm{Kn}=0.1$

$\begin{array}{lll}\text { (a) } \mathrm{z}^{+}=7.29 \mathrm{E}-4 & \text { (b) } \mathrm{z}^{+}=7.72 \mathrm{E}-3 & \text { (c) } \mathrm{z}^{+}=1.92\end{array}$

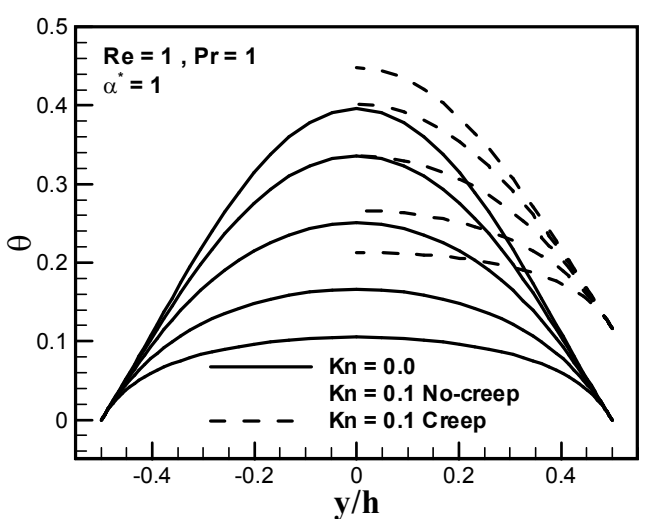

Fig. 12. Axial variation of non-dimensional temperature profiles in the symmetry plane of a square microchannel for $\mathrm{Re}=1$ in slip and no-slipflows: $z^{+}=z /\left(D_{h} \operatorname{Re}\right)=0.051,0.21,0.40$ and 2.00 with increasing $\theta$, respectively.

For such a case, temperature jump is only a function of the Knudsen number.

The maximum temperature difference $\Delta \theta$ between the core and the immediate vicinity of the wall is about 0.33 for $\mathrm{Kn}=0.1$, while this value increases to about 0.4 for $\mathrm{Kn}=0$. Apparently, at $\mathrm{Re}=1$, thermal creep does not affect the temperature distributions in the flow direction noticeably. However, at $\operatorname{Re}=0.1$, the temperature field is significantly influenced by thermal creep as shown in Fig. 13. The same flow conditions and axial locations as those in Fig. 8 are used here. It is seen that the uniform inlet temperature profile undergoes a continuous transformation along the channel until it reaches its maximum value in the center corresponding to the flat velocity profile in Fig. $8 \mathrm{~b}$. Farther down stream, the maximum non-dimensional temperature decreases gradually approaching its fixed fully developed profile at about $z^{+}=50$. Again, the symmetry plane is considered and the local wall temperature has been used as $T_{r e f}$.

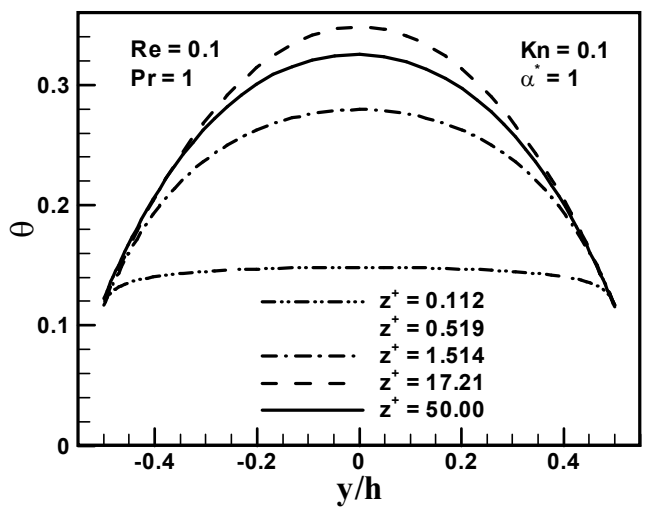

Fig. 13. Axial variation of non-dimensional temperature profiles in the symmetry plane of a square microchannel for $\mathrm{Re}=0.1$ and $\mathrm{Kn}=0.1$.

The circumferentially-averaged local heat transfer coefficient for the peripherally uniform heat flux boundary condition is defined as:

$N u=\frac{h_{x} D_{h}}{k}=\frac{q^{\prime \prime} D_{h}}{k\left(T_{w}-T_{m}\right)}$

where $T_{m}$ is the bulk mean temperature, and $T_{w}$ is the cross-sectional averaged wall temperature defined as:

$T_{w}=\frac{1}{P} \int_{S} T_{w, l} d s, \quad T_{m}=\frac{1}{A W_{i}} \int_{A} T \vec{V} \cdot d \vec{A}$

where $P$ is the perimeter, $T_{w, l}$ is the local wall temperature, $d s$ is a circumferential differential element and $A$ is the cross sectional area.

Figure 14 shows the axial variations of the heat transfer coefficients in a square microchannel for $\operatorname{Re}=1$. It is seen that the rarefaction effects dramatically reduce the heat transfer coefficient in the entrance region. There are two opposing effects regarding heat transfer. First, enhanced advection in the immediate vicinity of the wall due to velocity slip tends to increase heat transfer, and second, temperature jump at the wall which acts similar to a contact resistance tends to reduce heat transfer. The net effect is a reduction in heat transfer since the temperature jump effects outweigh the enhanced advection effects. Unlike the friction coefficient, the inclusion of thermal creep, which further enhances advection, does not appear to affect the heat transfer coefficient significantly.

Fully developed values of the Nusselt number for slipflow at $\mathrm{Re}=1$ are shown in Fig. 15 for square and rectangular microchannels with aspect ratios of 1 and 0.5 . For the case of fluid heating, thermal creep is expected to increase the heat transfer coefficient. Yet this effect is fairly small at $\operatorname{Re}=1$ for both aspect ratios. 


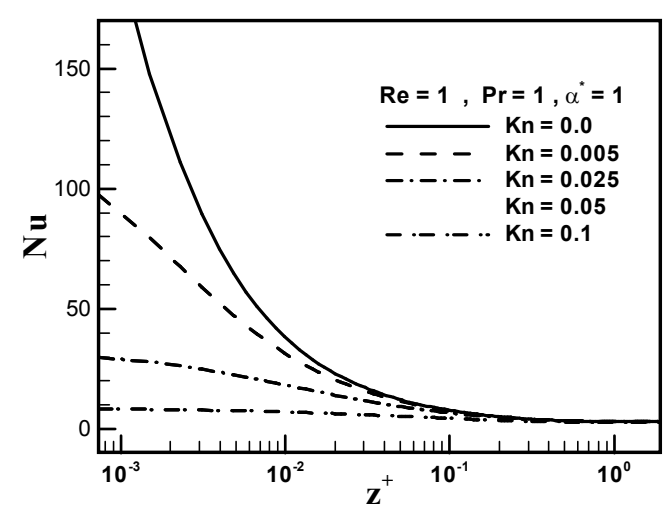

Fig. 14. Axial variation of the heat transfer coefficient for flow in a square microchannel at $\mathrm{Re}=1$ and various $\mathrm{Kn}$.

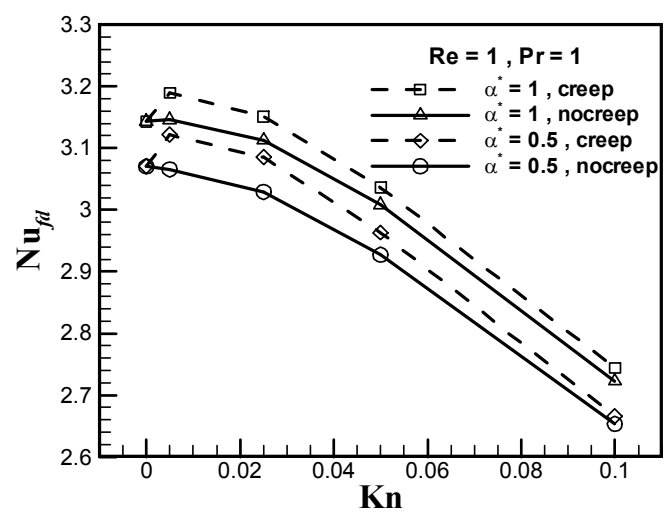

Fig. 15. Rarefaction and thermal creep effects on fully developed heat transfer coefficients

As indicated before, the velocity and temperature fields are very significantly influence by thermal creep at lower Reynolds numbers, and therefore, it is useful to compare the axial variation of $\mathrm{Nu}$ at two different Reynolds numbers as shown in Fig. 16, where the heat transfer coefficients are plotted for flow in a square microchannel at $\operatorname{Re}=0.1$ and 1 . At lower Reynolds numbers, the increase in advection effects near the wall due to thermal creep in addition to the decrease in temperature jump (because of flatter temperature profiles) results in a considerable increase in the Nusselt number along the microchannel, such that, an increase of about $39 \%$ in the heat transfer coefficient is observed in the fully developed region at $\mathrm{Re}=0.1$ as compared to its counterpart at $\mathrm{Re}=1$.

\section{CONCLUSIONS}

In this study, rarefaction and thermal creep effects on the flow and thermal development in rectangular microchannels are numerically examined in the slip and continuum flow regimes, $\mathrm{Kn} \leq 0.1$. Different channel aspect ratios at Reynolds numbers of 0.1 and 1 with $\mathrm{Pr}$ $=1$ are considered for a prescribed heat flux at the walls. Developing velocity profiles and thermal patterns in the entrance region are presented in detail. The effects of thermal creep, which are directly related to the applied heat flux, are studied on the key flow parameters including the friction and heat transfer coefficients. Dramatic reductions in the friction coefficient are observed in the entrance region due to rarefaction, which are enhanced by the effects of thermal creep.

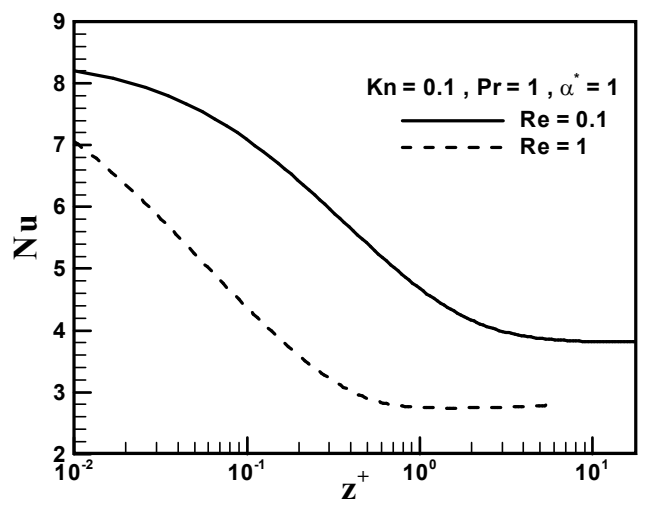

Fig. 16. Comparison of Nusselt number variation in a square microchannel at $\operatorname{Re}=0.1$ and 1 .

Besides the axial temperature gradient at the wall, thermal creep effects are also related to the fluid heating or cooling process. For the heating cases considered here, thermal creep increases velocity slip at the wall, and therefore, reduces further the friction coefficient and slightly enhances the heat transfer rates due to increased advection. For an identical heat flux applied to the microchannel walls, thermal creep effects become more pronounced at lower Reynolds number since it results in higher axial temperature gradients at the wall. Present results indicate that the flow and thermal fields are greatly influenced by thermal creep at $\mathrm{Re}=0.1$ for $\mathrm{Kn}=0.1$ Further investigations are needed to identify the thermal creep effects in flows where the fluid is being cooled.

\section{ACKNOWLEDGEMENTS}

The financial support of the Natural Sciences and Engineering Research Council of Canada and the Ferdowsi University of Mashhad are gratefully acknowledged.

\section{REFERENCES}

Bao, F. and J. Lin (2008). Burnett simulation of gas flow and heat transfer in micro Poiseuille flow. Int. J. Heat and Mass Transfer 51, 4139-4144.

Cetin, B., A.G. Yazicioglu and S. Kakac (2008). Fluid flow in microtubes with axial conduction including rarefaction and viscous dissipation. Int. Comm. in Heat and Mass Transfer 35, 535-544.

Chorin, A.J. (1968). Numerical solution of the NavierStokes equations. Math. Comput. 22, 745-762.

Dwyer, H.A. (1989). Calculation of droplet dynamics in high temperature environments. Prog. Energy Combust. Sci. 15, 131-158.

Ghahremani, A.R., S. Safari Mohsenabad and M. Behshad Shafii (2008). Analytical solution for compressible gas flow inside a two-dimensional Poiseuille flow in microchannels with constant heat flux including the creeping effect. Proceedings of 
World Academy of Science, Engineering and Technology 33, 143-147.

Hooman, K. (2008). A superposition approach to study slip-flow forced convection in straight microchannels of uniform but arbitrary crosssection. Int. J. Heat and Mass Transfer 51(15-16), 3753-3762.

Kuddusi, L. and E. Çetegen (2007). Prediction of temperature distribution and Nusselt number in rectangular microchannels at wall slip condition for all versions of constant heat flux. Int. J. Heat and Fluid Flow 28, 777-786.

Méolans, J.G. and I.A. Graur (2008). Continuum analytical modeling of thermal creep. European Journal of Mechanics - B/Fluids 27(6), 785-809.

Morini, G.L. and M. Spiga (1998). Slip-flow in rectangular microtubes. Microscale Thermophys. Engrg. 2, 273-282.

Morini, G.L., M. Spiga and P. Tartarini (2004). The rarefaction effect on the friction factor of gas flow in microchannels. Superlattices and Microstructures 35, 587-599.

Niazmand, H., M. Renksizbulut and E. Saeedi (2008). Developing slip-flow and heat transfer in trapezoidal microchannels. Int. J. Heat Mass Transfer 51(25-26), 6126-6135.

Renksizbulut, M. and H. Niazmand (2006). Laminar flow and heat transfer in the entrance region of trapezoidal channels with constant wall temperature. J. Heat Transfer 128, 63-74.

Rij, J.V., T. Harman and T. Ameel (2007). The effect of creep flow on two-dimensional isoflux microchannels. Int. J. Thermal Sciences 46, 10951103.

Sazhin, O., A. Kulev, S. Borisov and S. Gimelshein (2008). Numerical analysis of gas-surface scattering effect on thermal transpiration in the free molecular regime, Vacuum 82 20-29.

Shah, R.K. and A.L. London (1978). Laminar flow forced convection in ducts. Academic Press Inc., London.

Spiga, M. and G.L. Morini (1998). The developing Nusselt numbers for slip flow in rectangular duct. Int. J. Heat and Mass Transfer 41, 2799-2807.

Tunc, G. and Y. Bayazitoglu (2002). Heat transfer in rectangular microchannels. Int. J. Heat and Mass Transfer 45, 765-773. 